\title{
A Mental Model for a Rhetorical Arguer
}

\author{
Floriana Grasso (floriana@ csc.liv.ac.uk) \\ Department of Computer Science \\ The University of Liverpool \\ Liverpool, UK
}

\begin{abstract}
This paper proposes a general mental model for an agent capable to engage in rhetorical argumentation. The model is grounded in works in the philosophy of mind, and is inspired by research in contextual reasoning. The issues that are dealt with in this paper are mainly ontological, as we leave aside the problem of knowledge representation and reasoning, and concentrate on the conceptualisation of the model.
\end{abstract}

\section{Introduction}

We seek to build artificial agents able to engage in rhetorical argumentation. By rhetorical argumentation, in a tradition going back to Aristotle, we intend argumentation:

1. that is mainly based on evaluative judgements, rather than demonstrative proofs;

2. that puts, as a consequence, a great emphasis to the audience, and how arguments are perceived;

3. that relies heavily on the linguistic style of the presentation of the argument to the audience.

In this endeavour, we are grounded, as a point of departure, on the New Rhetoric (Perelman and OlbrechtsTyteca, 1969), an important theory in the philosophy of argument. We have presented in other works our characterisation of this theory, dealing with the issues of rhetorical reasoning, rhetorical schemata, dialogue games, and the description of an agent architecture for generating rhetorical natural discourse, notions which we have evaluated in a health promotion scenario (Grasso, 1998; Grasso et al., 2000; Grasso, 2002), and which we will not repeat here. Conversely, we deal, in this paper, with the challenge of representing the mental state of an agent involved in a rhetorical argumentation scenario. We do this from an ontological, rather than operational, point of view, as we describe a general model of what should be included in the mental model, leaving aside issues of reasoning with and about mental states.

\section{Framing the Problem}

In a view that has been embraced for a long time in AI, inspired by seminal works in the philosophy of mind, mental states are usually seen as relationships between an agent and a "mental object" (Russell and Norvig, 2003). The Russellian term "propositional attitude" has often been used to express this notion, referring to a sentence expressing a ternary relation between an agent, an attitude verb and a "that-clause" :

$$
\underbrace{\text { Floriana }}_{\text {Agent }} \underbrace{\text { believes }}_{\text {Verb att }} \underbrace{\text { that Liverpool is rainy. }}_{\text {that }- \text { clause }}
$$

In order to be able to argue rhetorically, the mental model of an agent should account for a variety of attitude verbs, and therefore of propositional attitudes. However, the AI coverage of what can be counted as an attitude verb has been far from comprehensive. In an interesting review (Shoham and Cousins, 1994) four categories of attitudes are identified:

1. informational attitudes, including mainly "knowledge" and "belief", but also "awareness", "doubt", etc.

2. motivational attitudes, concerning what drives an agent's action, for instance: goal, plan, intention, want, desire, choice, commitment.

3. social attitudes, concerning an agent's rights and obligations, when "living" in a society of agents.

4. emotional attitudes, concerning various extra-rational factors.

The informational attitudes are by far the most studied and understood (Fagin et al., 1996). The motivational category has been mostly studied under the umbrella of the Belief-Desire-Intention (BDI) model of agenthood (Rao and Georgeff, 1991). The social category has been almost exclusively investigated within the deontic logic paradigm. As to emotional attitudes, these have recently attracted the attention of the community working on the

\footnotetext{
${ }^{1}$ There has been much debate on the nature of such " $\mathrm{t}$ clauses", and some authors (Castañeda, 1975, most notably) have observed that one should distinguish between propositions (as in "I believe X") and practitions (as in "I intend to do X"). We adopt a non-sentential view and we assume that practitions can indeed be considered that-clauses (oversimplifying, one might rewrite the example as "I intend that I do X"). This view is largely accepted in the AI modelling of artificial agents (Wooldridge, 2000), when one would write $B E L(x)$ and $I N T E N D(x)$ similarly.
} 
"believability" characteristics of agenthood (Cañamero, 1998, for example). This means that important questions, for the model of a rhetorical arguer, like: "what it is like to 'remember' something?", or "what is the exact difference between 'assuming', 'believing' and 'supposing'?", or "how to represent 'prejudices' as opposed to 'beliefs'?" have not been answered satisfactorily.

If the model of the single agent's mental state is important, in rhetorical argumentation it is even more fundamental for an agent to represent the interlocutor's beliefs, as "In argumentation, the important thing is not knowing what the speaker regards as true or important, but knowing the views of those he is addressing" (Perelman and Olbrechts-Tyteca, 1969, § 5). Moreover, in the Gricean view of communication that argues it is necessary that the interlocutors share a common ground of beliefs, and know that they share them, two agents, A and B, should also be able to reason about mutual beliefs. This is what is believed at different "nesting levels", that is what:

1. A believes

2. B believes

3. A believes that B believes

4. B believes that A believes

5. A believes that $\mathrm{B}$ believes that $\mathrm{A}$ believes

6. B believes that A believes that B believes

etc. ad infinitum (Clark and Carlson, 1982). In AI systems, the problem of infinite recursion has been either solved directly, by generating "belief nestings" as needed (Ballim and Wilks, 1991, for example, adopt this view) or addressed by using an intuition, coming from the philosophy of mind, that agents have a "mutual belief induction schema", a sort of pre-compiled attitude that can be activated whenever agents assume they share some common ground. Computationally this means that, as soon as agreement is assumed on a particular level of belief nesting, all subsequent levels are considered equivalent. This is particularly appropriate when cooperation is assumed among agents: it has been noted (Taylor et al., 1996) the "apparent irrelevance" of representing deeply nested beliefs, for if no deception is expected or allowed, only a few levels of nesting (three, in fact, they propose) is needed to completely model a dialogue. Whatever the solution, it is clear that mutuality is a crucial concept for the model of a conversational agent.

\section{A "Rhetorical" Mental Model}

We describe here a very general model for an agent able to undertake rhetorical argumentation. The model is meant to be as non committing as possible, as we discuss what we believe is important to include, rather than try and solve issues of representation and reasoning. We discuss the model by considering the three issues we mentioned above as fundamental to the task: how to model the mental state, how to account for multiple attitudes, and how to represent mutuality.

\section{Representing Mental Attitudes}

We would like our agents to be able to reason about any mental attitude. We do not wish to state in advance the types of mental states an agent can be in, like, for instance, in the BDI framework, but we will simply write:

$$
\beta_{a}(x)
$$

to mean that agent $a$ is in the propositional attitude of $\beta$ towards the state of affair $x$. For instance, to express that Agent $A$ believes $X$ we will write: $B E L_{A}(X)$.

We expect our agents to show informational, motivational, social and emotional attitudes as needed by the argumentation, and indeed we expect rhetorical argumentation to make use to a great extent of a variety of attitude nuances. However, we do not propose, in this work, $a$ theory of mental attitudes: how they come to be, how they change, how they influence each other. While this may appear disappointing, it is also true that the only reasonable way to approach this problem is by investigating each and any of the mental attitudes with as much effort as there has been in the epistemological/informational attitudes analysis, and this is definitely outside the scope of our work.

We propose, however, a general framework to represent mental attitudes, which is inspired by work on contextual reasoning (Benerecetti et al., 2000). The intuition of contextual reasoning is that agents reason locally: they are not very likely to need their complete mental state to solve a particular reasoning task at hand, but they isolate a "box", a context, that, while containing all necessary information, is more manageable. Of particular interest to us is the notion of MultiContext systems (Giunchiglia and Bouquet, 1998): this adds, to the above mentioned locality assumption, the compatibility assumption that all contexts have to be related in some way in the agent's mind. Therefore, in addition to the rules which are valid within each context, and which might be of different nature, bridge rules can be used to reason across contexts. More formally, a context is a triple: $\langle L, \Omega, \Delta\rangle$ where $L$ is a language (e.g. first order logic), $\Omega$ is the set of axioms of the context and $\Delta$ is the set of inference rules that applies in the context. A MultiContext systems is a pair: $\langle C, B\rangle$ where $C$ is the set of all contexts, and $B$ the set of bridge rules. A bridge rule is in turn a rule of the type:

$$
c_{1}: \phi_{1} \ldots c_{n}: \phi_{n} \rightarrow c_{n+1}: \phi_{n+1}
$$

where $c_{i}: \phi_{i}$ means that formula $\phi_{i}$ is true in context $c_{i}$.

We see the agent's mental state as a set of "attitude contexts", where different contexts are used to represent different mental attitudes. An attitude context contains, for the single agent, not only the objects of that particular attitude, but also the reasoning rules that the agent uses to manipulate the propositional attitudes in the "box". This allows to postulate that different attitudes can have different reasoning styles. Principles to characterise and diversify attitudes (Barwise and Perry, 1983, for example, propose 8 principles to this aim) could be an example of rules internal to a context. In addition to internal rules, we also assume that the agent has a set of bridge rules, that allow to ascribe new propositional attitudes of a sort from others of different sorts. Similar to what 
has been hypothesised for intentions and beliefs (Cohen and Levesque, 1990), we can therefore imagine an agent having rules to decide, for instance, when a doubt can become a belief, and viceversa, or when fear leads to intention to act, and so on. Again, we do not want to make assumptions on how these bridge rules may look like, nor on their philosophical and psychological plausibility, and we leave this issue to further works. We therefore propose the following:

Definition 1 (Mental State) A mental state for a rhetorical arguer $\mathcal{M}$ is a multicontext system:

$$
\mathcal{M}=\langle B, R\rangle
$$

where $B$ is a set of attitude contexts, and $R$ is a set of bridge rules among the attitude contexts. An attitude context $\beta \in B$ is a triple:

$$
\beta=\langle\lambda, \alpha, \varrho\rangle
$$

where $\lambda$ is a language, $\alpha$ a set of axioms in $\lambda$ and $\varrho$ a set of inference rules internal to $\beta$.

As unique exception to generality, among the possible mental attitudes we see the attentional stance ("agent $\mathrm{A}$ has in focus that $\mathrm{X}$ ") and the evaluative stance ("agent $\mathrm{A}$ values $\mathrm{X}$ ") as something different than a propositional attitude. While they might be considered as contexts, we prefer to see them as meta reasoning abilities of the agent, as we see agents "use" them differently. In fact, the attentional attitude is something which makes sense only while the conversation is active. It is therefore more volatile than the other propositional attitudes, that are expected to be a property of the agent, not of the conversation, and will last after the conversation has terminated. Indeed, one can consider the focus as the subset of the mental state of the agent which is "active" at a particular time. Strictly speaking, it should be a multicontext containing subsets of the attitude contexts that are relevant at that moment. As to the evaluative attitude, we believe, with others (Galliers, 1992, most notably), this is best be seen as a "label" that can be associated to other mental attitudes (Grasso, 2002).

\section{Representing Mutuality}

We have discussed above the tension between computational systems implementing the "infinitely complete" version of mutuality, and those which adopt the point of view that there must be a "schema", a way to collapse the notion of mutuality from a certain level on, and just assume the agent have "reached" that level. We tend to agree with the latter view. The problem for us is then shifted to when we can assume mutuality has been reached, that is which is the ideal number of nesting levels that are needed for rhetorical argumentation.

In a situation of cooperative dialogue, no matter whether it is oriented to a collaborative or competitive task, or whether it is symmetrical with respect to the agents' role, agents are only interested in communicating what they believe, and assume their opponent does the same. This is the simplest communicative situation, and some authors (Taylor et al., 1996; Kobsa, 1990) argue that three levels of belief nesting are sufficient to represent $\mathrm{it}^{2}$, where the third level is really only needed to deal with misconceptions.

If one needs to model deceptive communication (Lee and Wilks, 1997), or communication where it is needed to "plan for plan recognition" (Taylor et al., 1996), that is to figure out what the other agent's plan might be, deeper nesting levels are needed. It is however difficult to imagine real life situations where more than a few more levels of nesting need to be added, as humans would tend to lose track of the connections.

But what are the characteristics of our arguing agents? Can they be defined as cooperative?

We would not want to go as far as saying that some sort of deception is in a sense inherent in the definition of rhetorical argumentation, for it is precisely this misconception that has associated to "rhetorical discourse" a negative connotation over the centuries. We have however to concede that the fact that a rhetorical argument is based on the audience's beliefs, and not on what the arguer really thinks on the matter, needs some of the devices of deception. In particular, when the arguer makes a standpoint which is only a projection of the audience's mind, like saying Eating fruit is important because it helps slimming to an audience who gives high value to being slim, the audience might ascribe this belief to the speaker, who on the other hand might have little interest in fitness. The ascription may well be just "for the sake of conversation" (Stalnaker, 1974), as the audience may not necessarily believe that the speaker believes that slimming is important. But such ascription is needed to create the grounds on which subsequent arguments can be discussed, evaluated, and backed or attacked.

So what do we need our agent to reason about? Let us consider the attitude of believing. We definitely need an agent to reason about private beliefs. We need the agent to reason about beliefs of other agents. And we need mutual beliefs. However, mutual beliefs depends on the interlocutor: agent $\mathrm{A}$ can reason about what is mutually believed with agent $\mathrm{B}$, which may be different from what $\mathrm{A}$ believes is mutually believed with agent $\mathrm{C}$. We represent this by creating separate environments in agent A's state of mind: what A believes that B believes that is mutually believed, what $\mathrm{A}$ believes that $\mathrm{C}$ believes that is mutually believed, and so forth.

But this still does not capture the expressiveness we would like. We have maintained that rhetorical argumentation is not about absolute facts, but about what agents perceive the reality is. It has therefore little sense to say that agents $\mathrm{A}$ and $\mathrm{B}$ have a mutual belief that $X$, as in this case $X$ would be a given fact. What we really would like to represent is that agents $\mathrm{A}$ and $\mathrm{B}$ have a mutual belief that someone believes $X$, where "someone", in the discussion between $\mathrm{A}$ and $\mathrm{B}$, can be either of them. We

${ }^{2}$ (1) What A believes, (2) what A believes that B believes, and (3) what is mutually believed. 
see, in other words, the third belief nesting mentioned by (Taylor et al., 1996; Kobsa, 1990) as in fact a "double" environment, which depends on the interlocutor whose standpoint is considered.

This allows to represent situations in which an agent realises that what the other has perceived from the conversation is not exactly what is in fact believed, because of a misconception, or because of a statement of a "belief for the sake of conversation". An agent A saying "eating fruit helps slimming" may assume to be ascribed by another agent B the belief that "slimming is important", even if this is not the case:

1. A believes that not X;

2. A believes that B believes that it is mutually believed that A believes that $\mathrm{X}$;

Symmetrically, agent A can ascribe a "belief for the sake of conversation" to agent B (including it in the mutual belief section) while not really ascribing the belief to $\mathrm{B}$ :

1. A believes that $\mathrm{B}$ believes that not $\mathrm{X}$;

2. A believes that B believes that it is mutually believed that $\mathrm{B}$ believes that $\mathrm{X}$.

Therefore the situation of "mutual belief that X", as envisaged before (Clark and Carlson, 1982), corresponds to the set of beliefs (from agent A's point of view):

1. A believes that $X$;

2. A believes that $\mathrm{B}$ believes that $\mathrm{X}$;

3. A believes that B believes that it is mutually believed that A believes that X;

4. A believes that B believes that it is mutually believed that $\mathrm{B}$ believes that $\mathrm{X}$.

\section{Mutuality across Attitudes}

We assume that mutuality can be expressed for several attitudes (though we are prepared to accept that for some attitudes it will have less sense). For instance, one can imagine to define mutual desire, as a paraphrase of the definition of mutual belief, as:

1. A desires that $p$.

2. B desires that $p$.

3. A desires that $\mathrm{B}$ desires that $p$.

4. $\mathrm{B}$ desires that $\mathrm{A}$ desires that $p$.

5. A desires that $\mathrm{B}$ desires that $\mathrm{A}$ desires that $p$.

6. $\mathrm{B}$ desires that $\mathrm{A}$ desires that $\mathrm{B}$ desires that $p \ldots$

This definition usefully implements co-ordination of action among agents (Clark and Carlson, 1982, give the example of the violin duet, where the two players must start simultaneously) in BDI contexts where desire leads to intention (and therefore action).

We assume, however, that mutual attitudes are expressed towards agent's attitudes, and not mere facts, that is we want to represent that it is "mutually believed that A believe X", or that is it "mutually desired that A believes X", and so on. We assume, in other words, a generic multi attitude scenario, where inner attitudes, and mutual attitudes, can be defined as needed.

Figure 1 shows a graphical representation of nesting levels in a generic multi attitude scenario, where:

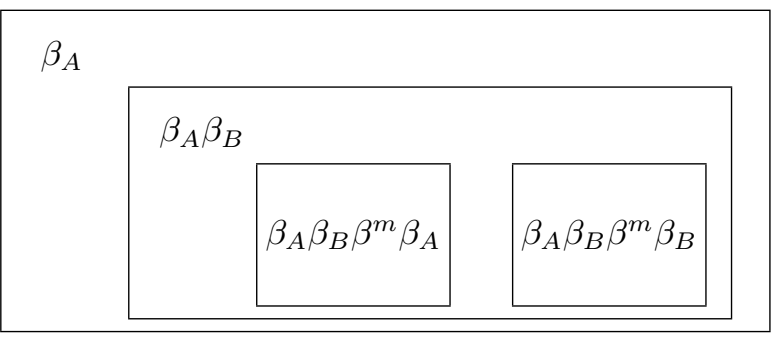

Figure 1: Model of Belief Nestings

$\beta_{A}$ is agent $A$ 's own mental state ( $A$ believes $\mathrm{X}$, or wants $\mathrm{Y}$ or doubts that $\mathrm{Z}$, etc.)

$\beta_{A} \beta_{B}$ are attitudes ascribed by $A$ to agent $B$ ( $A$ believes that $B$ believes $\mathrm{X}$, or $A$ believes that $B$ wants $\mathrm{Y}$, etc).

$\beta_{A} \beta_{B} \beta^{m} \beta_{A}$ represents $A$ 's view on $B$ 's view on what mutually happens "as regard to $A$ " (e.g. $A$ believes that $B$ wants that it is mutually believed that $A$ believes $\mathrm{X})$.

$\beta_{A} \beta_{B} \beta^{m} \beta_{B}$ represents $A$ 's view on $B$ 's view on what mutually happens "as regard to $B$ " (e.g. $A$ believes that $B$ wants that it is mutually believed that $B$ believes X).

Mutuality as Attitude Contexts Going back to the problem of representing the mental model, we consider the levels of nesting as if they were different propositional attitudes (Thomason, 2000). In other words, we assume that, for instance, "believing" and "believing that agent X believes" are two different attitude contexts, and we assign them different "boxes". This makes sense if it is noted that agents may use reasoning rules, when trying to put themselves in other agents' shoes, which may well be different than those used when reasoning on their own mental state. Moreover, we can assume these reasoning rules are dependent on the particular agent they reason upon, so "believing that agent $\mathrm{X}$ believes" may work differently than "believing that agent Y believes". As described above, bridge rules are used to reason across contexts, allowing, for example, to conclude something on what another agent believes on the basis on one's own beliefs.

In this hypothesis, the number of attitude contexts that there are in an agent's mental state depends on the number of agents to reason about, and the number of propositional attitudes to consider. For instance, if two attitudes only are considered (say "belief" and "want") in a two agent ( $\mathrm{A}$ and $\mathrm{B}$ ) scenario, agent $\mathrm{A}$ has a context for each private mental attitude:

1. what A believes;

2. what A wants.

Then, agent A might be in one of the two attitudes towards any attitude of agent B: 


\begin{tabular}{|c|c|c|c|c|c|c|c|c|c|}
\hline & & \multicolumn{4}{|c|}{ Agent A } & \multicolumn{4}{|c|}{ Agent B } \\
\hline Case & Agent A says & $\beta_{A}$ & $\beta_{A} \beta_{B}$ & $\beta_{A} \beta_{B} \beta^{m} \beta_{A}$ & $\beta_{A} \beta_{B} \beta^{m} \beta_{B}$ & $\beta_{B}$ & $\beta_{B} \beta_{A}$ & $\beta_{B} \beta_{A} \beta^{m} \beta_{B}$ & $\beta_{B} \beta_{A} \beta^{m} \beta_{A}$ \\
\hline $\mathrm{a} 1$ & I believe X & $\mathrm{X}$ & & $\bar{X}$ & & & $\mathrm{X}$ & & $\mathrm{X}$ \\
\hline a 2 & You believe $X$ & & $\mathrm{X}$ & & & & & $\mathrm{X}$ & \\
\hline $\mathrm{b}$ & I believe X & $\mathrm{X}$ & & $\mathrm{X}$ & & & not X & & $\mathrm{X}$ \\
\hline $\mathrm{c}$ & Ibelieve X & $\operatorname{not} X$ & & $\mathrm{X}$ & & & not X & & $\mathrm{X}$ \\
\hline $\mathrm{d}$ & I believe X & $\operatorname{not} \mathrm{X}$ & & $\mathrm{X}$ & & & $\mathrm{X}$ & & $\mathrm{X}$ \\
\hline
\end{tabular}

Figure 2: Communication types

3. what A believes that B believes;

4. what A believes that B wants;

5. what A wants that B believes;

6. what $\mathrm{A}$ wants that $\mathrm{B}$ wants.

Finally, agent A needs to store mutual beliefs and wants, with different combinations:

7. what A believes that $\mathrm{B}$ believes that is mutually believed that $\mathrm{B}$ believes;

8. what A believes that B believes that is mutually believed that B wants;

9. what $\mathrm{A}$ believes that $\mathrm{B}$ believes that is mutually wanted that B believes;

20. what A wants that B wants that is mutually believed that B wants;

21. what A wants that B wants that is mutually wanted that B believes;

22. what A wants that B wants that is mutually wanted that B wants.

23. what $\mathrm{A}$ believes that $\mathrm{B}$ believes that is mutually believed that A believes;

38. what A wants that B wants that is mutually wanted that A wants.

In general, for two agent scenarios, with $n$ attitudes, the number of attitude contexts for one agent is:

$$
n+n^{2}+2 n^{4}
$$

If $k$ agents are to be considered, and we assume agents do not have a complete picture of what other agents think of other agents' mental state (that is we do not represent, say, that agent $F$ believes that agent $\mathrm{G}$ believes that agent $\mathrm{H}$ believes... ${ }^{3}$ ), the number of attitude contexts for one agent is:

$$
n+k\left(n^{2}+2 n^{4}\right)
$$

\section{Use of Contexts in Rhetorical Dialogues}

We assume that an agent, A, when performing a dialogue move, is confident in the "success" of the move, and therefore it stores in the self related mutual attitude context $\left(\beta_{A} \beta_{B} \beta^{m} \beta_{A}\right)$ that the move has taken place. The

\footnotetext{
${ }^{3}$ We are not aware of any study of multiagent dialogues which takes this kind of situation into account, but we suspect that conclusions on the ability of humans to keep track of chains of beliefs can be made which are similar to those on infinitely nested mutual beliefs.
}

attitude invoked will depend on what the agent has expressed: whether a belief, or a wish etc. A similar assumption is made by the listening agent $\mathrm{B}$, that is it will assume $\mathrm{A}$ is confident of the success of its move, and will update the relevant context $\left(\beta_{B} \beta_{A} \beta^{m} \beta_{A}\right)$. Therefore, whatever is "said" is by default included in the appropriate mutuality contexts of the dialoguing agents. On the other hand, what the speaking agent's real point of view is, or what the listening agent really ascribes to the speaker after a communication, depends on the agents' conclusion from the appropriate bridge rules and will be stored in the "private" sections of their mental state.

Figure 2 represents how the various parts of the model of two agents, A and B, are updated after different sorts of communication, showing that the four sets proposed are necessary and sufficient to represent the different situations. The figure only considers one attitude (belief) for the sake of clarity, but this can be extended to multiattitude models. The communicated piece of information can be either sincere (cases a, b), or insincere (cases c, d), that it is not faithful to the actual agent's belief. When a communication is sincere, and is perceived as sincere, all the sections of the belief model of both agents contain the same piece of information (cases a). In cases in which either a misinterpretation, or a belief for the sake of conversation (or indeed a lie) occur, differences exist between the mutual and the private sections of both agents, where the mutual sections mirror what has been communicated, and the private ones express the beliefs which are actually held. It should be noticed that case (a) is the only situation in which the communication concerns a second level belief, that is a belief about the other agent's beliefs (in subcase $\mathrm{a} / 2$ ). We assume in fact that when agents talk about each other beliefs, only a straight, truthful communication can take place (one agent cannot say "I believe you believe X" if this is not the case). Similarly, we also note that we cannot represent more sophisticated styles of communication, such as irony, in which the speaker utters false statements with the assumption that the hearer will realise that. In both cases, in order to be able to represent this properly we would need extra levels of attitude nesting (Lee and Wilks, 1997). We can, however, represent situations of A and B "agreeing

\begin{tabular}{|c|c|c|c|c|}
\hline & $\begin{array}{c}\beta_{A} \\
\mathrm{X}\end{array}$ & $\begin{array}{l}\beta_{A} \beta_{B} \\
\operatorname{not} X\end{array}$ & $\begin{array}{c}\beta_{A} \beta_{B} \beta^{m} \beta_{A} \\
\mathrm{X}\end{array}$ & $\begin{array}{c}\beta_{A} \beta_{B} \beta^{m} \beta_{B} \\
\operatorname{not} \mathrm{X}\end{array}$ \\
\hline & $\begin{array}{c}\beta_{B} \\
\text { not X }\end{array}$ & $\begin{array}{c}\beta_{B} \beta_{A} \\
\mathrm{X}\end{array}$ & $\begin{array}{c}\beta_{B} \beta_{A} \beta^{m} \beta_{B} \\
\operatorname{not} \mathrm{X}\end{array}$ & $\begin{array}{c}\beta_{B} \beta_{A} \beta^{m} \beta_{A} \\
\mathrm{X}\end{array}$ \\
\hline
\end{tabular}
to disagree": 


\section{Conclusions}

We have explored, in this paper, the problem of representing the mental state of a rhetorical arguer, in the spirit of producing an ideal framework, rather than a specific, implementation oriented design for the agent's mental state. By drawing upon fundamental notions of philosophy of mind, and by analysing how these notions have been implemented, we have proposed a clear picture of what is needed to represent an agent able to argue rhetorically. From this, the reader interested in actually implementing the agent, can benefit from off-the-shelf formal models, whether from logic, probability or constraint reasoning, both to reason within the contexts and for implementing bridge rules. We will not choose a preferred one, and we leave the evaluation of which is the best approach out of the scope of this work.

\section{References}

Ballim, A. and Wilks, Y. (1991). Artificial Believers. Lawrence Erlbaum Associates.

Barwise, J. and Perry, J. (1983). Situations and Attitudes. MIT Press, Cambridge, MA.

Benerecetti, M., Bouquet, P., and Ghidini, C. (2000). Contextual reasoning distilled. Journal of Experimental and Theoretical Artificial Intelligence, 12(3):279-305.

Cañamero, D., editor (1998). Emotional and Intelligent: The Tangled Knot of Cognition. Papers from the 1998 AAAI Fall Symposium. AAAI Press.

Castañeda, H.-N. (1975). Thinking and Doing. Kluwer.

Clark, H. and Carlson, T. (1982). Speech Acts and Hearer's Beliefs. In Smith, N., editor, Mutual Knowledge, chapter 1, pages 1-36. Academic Press.

Cohen, P. and Levesque, H. (1990). Intention is Choice with Commitment. Artificial Intelligence, 42:213261.

Fagin, R., Halpern, J., Moses, Y., and Vardi, M. (1996). Reasoning about Knowledge. MIT Press, London.

Galliers, J. (1992). Autonomous Belief Revision and Communication. In Gärdenfors, P., editor, Belief Revision, pages 220-246. Cambridge University Press.

Gernsbacher, M. and Derry, S., editors (1998). Proceedings of the 20th Annual Meeting of the Cognitive Science Society. Lawrence Erlbaum Associates.

Giunchiglia, F. and Bouquet, P. (1998). A context based framework for mental representation. In (Gernsbacher and Derry, 1998), pages 392-397.

Grasso, F. (1998). Exciting avocados and dull pears: Combining behavioural and argumentative theory for producing effective advice. In (Gernsbacher and Derry, 1998), pages 436-441.
Grasso, F. (2002). Towards a framework for rhetorical argumentation. In Bos, J., Foster, M., and Matheson, C., editors, EDILOG'02: Proceedings of the 6th workshop on the semantics and pragmatics of dialogue, pages 53-60, Edinburgh.

Grasso, F., Cawsey, A., and Jones, R. (2000). Dialectical Argumentation to Solve Conflicts in Advice Giving: a case study in the promotion of healthy nutrition. International Journal of Human-Computer Studies, 53(6):1077-1115.

Kobsa, A. (1990). Modeling the User's Conceptual Knowledge in BGP-MS, a User Modeling Shell System. Computational Intelligence, 6:193-208.

Lee, M. and Wilks, Y. (1997). Eliminating Deceptions and Mistaken Belief to Infer Conversational Implicature. In Jokinen, K., Sadek, D., and Traum, D., editors, IJCAI 1997 Workshop on Collaboration, Cooperation and Conflict in Dialogue Systems.

Perelman, C. and Olbrechts-Tyteca, L. (1969). The New Rhetoric: a treatise on argumentation. University of Notre Dame Press, Notre Dame, Indiana.

Rao, A. and Georgeff, M. (1991). Modeling Rational Agents within a BDI-Architecture. In Allen, J., Fikes, R., and Sandewall, E., editors, Proceedings of the 2nd International Conference on Principles of Knowledge Representation and Reasoning (KR'91), pages 473-484. Morgan Kaufmann Publishers.

Russell, S. and Norvig, P. (2003). Artificial Intelligence: A Modern Approach. Prentice Hall, 2nd edition.

Shoham, Y. and Cousins, S. (1994). Logics of Mental Attitudes in AI, a Very Preliminary Survey. In Lakemeyer, G. and Nebel, B., editors, Foundations of Knowledge Representation and Reasoning, volume 810 of LNAI. Springer-Verlag.

Stalnaker, R. (1974). Pragmatic presuppositions. In Munitz, M. and Unger, P., editors, Semantics and Philosophy, pages 197-213. New York University Press, New York, NY.

Taylor, J., Carletta, J., and Mellish, C. (1996). Requirements for Belief Models in Cooperative Dialogue. User Modeling and User-Adapted Interaction, 6(1):23-68.

Thomason, R. (2000). Modeling the beliefs of other agents. In Minker, J., editor, Logic-Based Artificial Intelligence, pages 375-473. Kluwer Academic Publishers, Dordrecht.

Wooldridge, M. (2000). Reasoning About Rational Agents. MIT Press, Cambridge, MA. 